\title{
Sums of Exponentials Approximations for the Kohlrausch Function
}

\author{
R. S. Anderssen ${ }^{\mathrm{a}}$, M. P. Edwards ${ }^{\mathrm{b}}, \underline{\text { S. A. Husain }}^{\mathrm{c}}$ and R. J. Loy $^{\mathrm{d}}$ \\ ${ }^{a}$ CSIRO Mathematics, Informatics and Statistics, GPO Box 664, Canberra, ACT 2601 \\ ${ }^{\mathrm{b}}$ School of Mathematics and Applied Statistics, University of Wollongong, Wollongong, NSW 2522 \\ ${ }^{\mathrm{c}}$ Department of Mathematics, Faculty of Science, University of Brunei \\ ${ }^{\mathrm{d}}$ Department of Mathematics, Mathematical Sciences Institute, \\ Australian National University, Canberra, ACT 0200 \\ Email: Bob.Anderssen@csiro.au
}

\begin{abstract}
The mathematical foundation of many real-world problems can be quite deep. Such a situation arises in the study of the flow and deformation (rheology) of viscoelastic materials such as naturally occurring and synthetic polymers. In order to advance polymer science and the efficient manufacture of synthetic polymers, it is necessary to recover information about the molecular structure within such materials. For the recovery of such information about a specific polymer, it is necessary to determine its relaxation modulus $G(t)$ and its creep modulus $J(t)$. They correspond to the kernels of the Boltzmann causal integral equation models of stress relaxation and strain accumulation experiments performed on viscoelastic solids and fluids. In order to guarantee that the structure of such models is consistent with the conservation of energy, both the relaxation modulus and the derivative of the creep modulus must be completely monotone (CM) functions.
\end{abstract}

As well as the exponential function $\exp (-\alpha t)$ and Dirichlet series

$$
\sum_{n=1}^{\infty} a_{i} \exp \left(-\lambda_{i}\right), \quad 0<\lambda_{1}<\lambda_{2}<\cdots,
$$

with non-negative coefficients $a_{i}$, the set of CM functions includes many interesting examples such as the subclass

$$
\exp (-\dot{\theta}), \quad \dot{\theta}=\frac{d \theta}{d t}, \quad \theta(0)=0, \quad \theta(t) \in \mathrm{CM}
$$

An important practical example in this subclass are the Kohlrausch (Williams Watts; stretched exponential) functions

$$
\exp \left(-\alpha t^{\beta}\right), \quad 0<\beta<1 .
$$

Such functions arise in a broad spectrum of applications and have important mathematical properties Anderssen et al. (2004) (e.g. the Weibull cumulative probability distribution).

Because of their importance in applications, there is a need to have accurate approximations for it, so that its properties can be approximated. For example, even though the relaxation and creep modulii $G(t)$ and $J(t)$ of linear viscoelasticity are known to satisfy the interconversion equation

$$
\int_{0}^{t} G(t-\tau) J(\tau) d \tau=\int_{0}^{t} J(t-\tau) G(\tau) d \tau=t
$$

the specific form of $J(t)$ is unknown when $G(t)$ is a Kohlrausch function. Theoretically, it is known that $J(t)$ must be a strictly monotonically increasing function.

Recently, by taking advantage of the Pollard (1946) result that a Kohlrausch function is the Laplace transform of a known stable distribution, Anderssen and Loy (2011) have shown how uniformly convergent sums of exponentials approximations for the Kohlrausch function can be constructed. In this paper, the derivation, significance and validation of such approximations are examined.

Keywords: Kohlrausch, complete monotonicity, sums of exponentials, Dirichlet series, interconversion 


\section{Complete Monotonicity and Kohlrausch Functions}

A $\left.C^{\infty}[0, \infty)\right)$ function $f:(0, \infty) \rightarrow[0, \infty)$ is completely monotone $(\mathcal{C} M)$ if it satisfies the rather innocuous conditions

$(-1)^{n} \frac{d^{n} f}{d x^{n}} \geq 0, \quad n=0,1,2, \ldots$

Widder (1946) gives an alternative definition: a $\left.C^{\infty}[0, \infty)\right)$ function $f:[0, \infty) \rightarrow[0, \infty)$ is completely monotone if it satisfies the condition (1) and is continuous at 0 . However, this aspect will not be pursued here. The importance of this family of functions is the following result of Bernstein (1928) that: a function $f:(0, \infty) \rightarrow[0, \infty)$ is $\mathcal{C} M$ if and only if there is a positive locally finite Borel measure $\mu$ on $(0, \infty)$ such that

$f(s)=\int_{0}^{\infty} \exp (-s t) d \mu(t), \quad s>0$.

The $\mathcal{C} M$ functions arise as the fundamental objects in a wide range of applications including

(i) The diesel spray problem. (Grinshpan et al. (2000)) Modelling the dynamics of diesel spray is fundamental to improving on current designs for diesel engines and on the efficency with which the fuel is burnt when it explodes sponstaneously under compression. The overall details are highly complex. However, insight is gain through the identification of simplifications which encapsulate the essential details. In the current situation, the simplification which has proved seminal is the assumption that the logarithmic derivative of the diesel parameter function behaves like a $\mathcal{C} M$ function.

(ii) The causal modelling of linear viscoelasticity. (Anderssen et al. (2008a,b)) The stress-strain measurements of linear viscoelastic materials take two forms:

(a) The measured stress $\sigma(t)$ in response to an applied strain rate $\dot{\gamma}(t)$ which is modelled causally as

$$
\sigma(t)=\int_{-\infty}^{t} G(t-\tau) \dot{\gamma}(\tau) d \tau, \quad G(t):=\text { the relaxation modulus. }
$$

(a) The measured strain $\gamma(t)$ in response to an applied stress rate $\dot{\sigma}(t)$ which is modelled causally as

$$
\gamma(t)=\int_{-\infty}^{t} J(t-\tau) \dot{\sigma}(\tau) d \tau, \quad J(t):=\text { the retardation (creep) modulus. }
$$

In order to ensure that conservation of energy is guaranteed, it is usually assumed that the relaxation modulus $G$ and the derivative $\dot{J}$ of the retardation modulus $J$ are $\mathcal{C} M$ functions.

(iii) Dielectric susceptability. The electric susceptibility $\chi_{E}$ measures how easily a dielectric material polarizes in response to an applied electric field. It, in turn, can be used to determine other electric properties of a material such as permittivity. The measurement process is causal and takes a form similar to that for the causal models of viscoelastic materials.

As well as the exponential function $A \exp (-\alpha t)$, it is common practice to choose the Kohlrausch function $A \exp \left(-\alpha t^{\beta}\right), 0<\beta<1$ as the model of the underlying $\mathcal{C} M$ behaviour. The popularity of the Kohlrausch function relates to the facts that

- it has a small (three) parameter representation,

- like for the Gompertz model (Osborne and Anderssen (2003)), the associated parameter identification for $A, \alpha$ and $\beta$ can be achieved using a double logarithmic transformation,

$$
y(t)=A \exp \left(-\alpha t^{\beta}\right) \quad \Leftrightarrow \quad A=y(0) \quad \Leftrightarrow \quad \ln (-\ln (y(t) / y(0))=\alpha+\beta t,
$$


- it yields a more comprehensive representation of $\mathcal{C} M$ behaviour that can be achived with a small number of exponential functions, and

- for small $\beta \sim 0$, it yields a good model for a switching process.

Further details about Kohlrausch functions can be found in Lindsey and Patterson (1980), Anderssen et al. (2004) and Macdonald (2006).

\section{The Sums of Exponentials Approximations}

There are situations where it would be useful if the Laplace transform of the Kohlrausch function was known analytically. Such a situation arizes in linear viscoelasticity. If the relaxation modulus $G$ is assumed to be a Kohlrausch function, then, in order to determine the correspond structure for the retardation modulus $J$, it is necessary to solve the interconversion equation (Anderssen et al. (2008a,b))

$$
\int_{0}^{t} G(t-\tau) J(\tau) d \tau=t
$$

Unfortunately, the Laplace transform of a Kohlrausch function is unknown in an explicit sense, though implicit definitions have been derived.

The alternative strategy is to approximate the Kohlrausch functions by a sum of functions for which the Laplace transform is known. The obvious choice is the exponential functions, for which there is a comprehensive literature about how to solve the interconversion equation when $G$ is given as a sum of exponential functions (Nikonov et al. (2005)).

Using non-constructive arguments, based on the classical results of Müntz, Liu (1999, 2001b) established conditions under which a completely monotone function could be approximated by a Dirichlet series with positive coefficients, and exploited this result to derive estimates of the relaxation spectrum of a linear viscoelastic material from creep measurements Liu $(1999,2001 \mathrm{a})$. Among other things, he proved "if a function can be approximated arbitrarily closely by Dirichlet series with nonnegative coefficients in supremum norm or $L_{p}$-norm, $1 \leq p<\infty$, then it must be completely monotonic".

As well as being important from a theoretical perspective, Liu's results open up the question about how such sums of exponentials should be constructed. Because of its practical importance in a wide range of applications (Macdonald (2006); Anderssen et al. (2004); Macdonald (1997); Lindsey and Patterson (1980)), the completely monotone Kohlrausch function represents a key candidate to examine. The starting point is Pollard's 1946 rigorous proof Pollard (1946) of the following Laplace transform identity for the Kohlrausch function

$\exp \left(-t^{\beta}\right)=\int_{0}^{\infty} \phi(\beta, p) \exp (-p t) d p, \quad 0<\beta \leq 1, \quad t \geq 0$,

where

$\phi(\beta, p)=\frac{1}{\pi} \int_{0}^{\infty} \exp (-p u) \psi(u) d u, \quad \psi(u)=\exp \left(-u^{\beta} \cos (\pi \beta)\right) \sin \left(u^{\beta} \sin (\pi \beta)\right)$.

The function $\phi(\beta, p)$, a stable distribution in the sense of Levy (Montroll and Bendler (1984); Feller (1966)), has a variety of useful summation and integral representations (Pollard (1946); Anderssen et al. (2004)). In particular, it is non-negative and has the following properties:

- For all $\beta$,

$$
\int_{0}^{\infty} \phi(\beta, p)=1
$$

- For each $\beta$, there exists a unique $\hat{p}_{\beta}$ such that

$$
\phi\left(\beta, \hat{p}_{\beta}\right)=\max _{p} \phi(\beta, p)=K_{\beta} .
$$


- It follows from the unimodality of $\phi(\beta, p)$ that, for a fixed $\beta$, there exists a unique $p_{\beta}$ such that $\phi(\beta, p)$ is strictly monotone decreasing for $p>p_{\beta}$.

It is clear from the structure of the right hand side of equation (3), because it already contains the exponential function as part of the integrand, some approximate utilization of the integral mean value theorem would generate sums of exponentials approximations. This is the strategy adopted and exploited by Anderssen and Loy (2011). They used the integral mean value theorem to construct a sum of exponentials approximation to the Kohlrausch function and to prove its uniform convergence. In this way, they derived the following family of approximations

Take a positive interger $N>1$. The identity (3) can be rewritten in the following compartmentalized form

$\exp \left(-t^{\beta}\right)=\Upsilon_{0}+\sum_{i=1}^{N} \int_{p_{i}}^{p_{i+1}} \phi(\beta, p) \exp (-p t) d p+\Upsilon_{\infty}$

where

$$
\begin{aligned}
0 & <p_{1}=p^{\#}(\beta)<p_{2}<\ldots<p_{N}<p_{N+1}=p^{\# \#}(\beta)<\infty, \\
p_{i+1}-p_{i} & =\frac{\left(p^{\# \#}(\beta)-p^{\#}(\beta)\right)}{N}, \quad i=1,2, \ldots, N,
\end{aligned}
$$

and, for a given $\epsilon>0, p^{\#}(\beta)$ and $p^{\# \#}(\beta)$ are chosen so that

$$
\begin{aligned}
\Upsilon_{0} & =\int_{0}^{p^{\#}(\beta)} \phi(\beta, p) \exp (-p t) d p<\epsilon, \\
\Upsilon_{\infty} & =\int_{p^{\# \#(\beta)}}^{\infty} \phi(\beta, p) \exp (-p t) d p<\epsilon .
\end{aligned}
$$

Because $\phi(\beta, p)$ is a non-negative function, the integral mean value theorem can be applied to the individual integrals in the representation (5) to yield

$$
\begin{aligned}
\exp \left(-t^{\beta}\right) & \approx \sum_{i=1}^{N} \exp \left(-p^{*}(i, t) t\right) \int_{p_{i}}^{p_{i+1}} \phi(\beta, p) d p \\
& =\sum_{i=1}^{N} a_{i}^{*} \exp \left(-p^{*}(i, t) t\right)
\end{aligned}
$$

where $p^{*}(i, t)$, with $p_{i}<p^{*}(i, t)<p_{i+1}$, is the corresponding mean value of $p$, and

$$
a_{i}^{*}=\int_{p_{i}}^{p_{i+1}} \phi(\beta, p) d p, \quad i=1,2, \ldots, N
$$

Using the following approximations for the mean values $p^{*}(i, t)$

$$
p_{i}^{*}=\frac{p_{i}+p_{i+1}}{2}, i=1,2, \ldots, N
$$

equation (6) becomes

$\exp \left(-t^{\beta}\right) \approx \sum_{i=1}^{N} a_{i}^{*} \exp \left(-p_{i}^{*} t\right)$ 


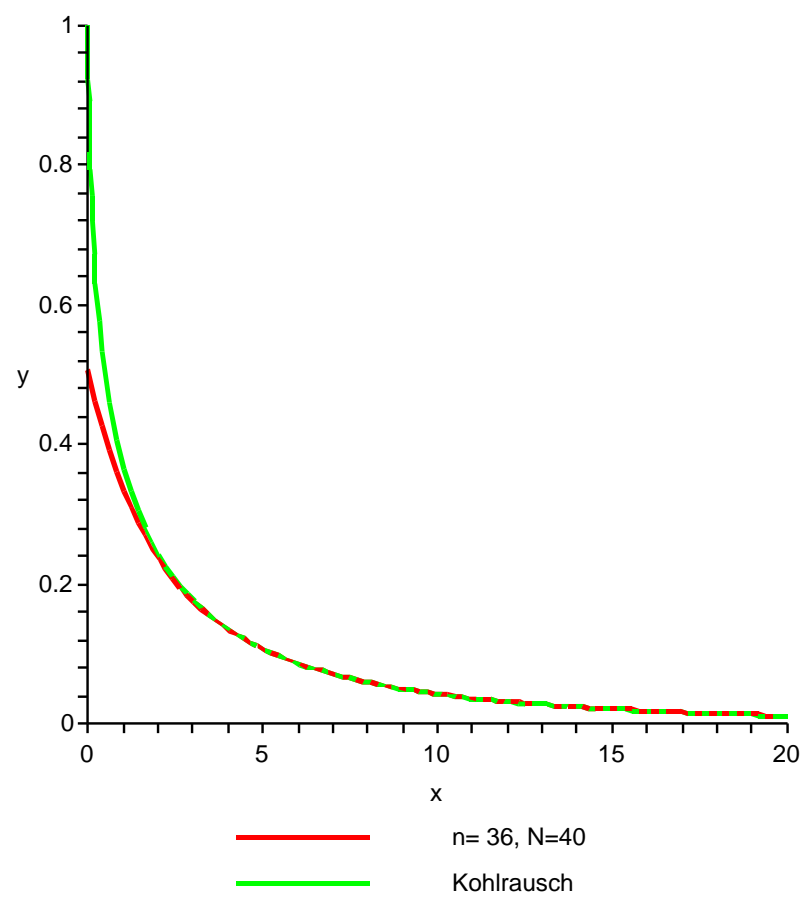

Figure 1: A plot in green of the Kohlrausch function $\exp \left(-t^{1 / 2}\right)$ and the approximation generated for $n=36$ and $N=40$.

\section{IMPLEMENTATION}

For the approximations (7), the key step is the evaluation of the coefficients $a_{i}^{*}$. A change in the order of the integration in the formula for $a_{i}^{*}$ and a little algebra yields

$a_{i}^{*}=\Psi\left(p_{i}\right)-\Psi\left(p_{i+1}\right), \quad \Psi(p)=\frac{1}{\pi} \int_{0}^{\infty} \frac{\psi(u)}{u} \exp (-p u) d u$

With $0<\beta<1$, the singularity in $\Psi(p)$ is integrable.

Because of the oscillatory term $\sin \left(u^{\beta} \sin (\pi \beta)\right)$ in $\psi(u)$, accurate evaluation of the $a_{i}^{*}$ must involve the careful cancellation of the positive and negative contributions. The extenuating factor is the decay associated with the $\exp (-p u)$ term, which implies that, for a given $p$, there exists a $\bar{u}$ such that the value of

$$
\int_{\bar{u}}^{\infty} \frac{\psi(u)}{u} \exp (-p u) d u
$$

is negligible.

However, in the special case when $\beta=1 / 2$, it can be shown (e.g. using Maple) that

$$
\Psi(p)=\operatorname{erf}\left(\frac{1}{2 \sqrt{p}}\right),
$$

and, hence, that the corresponding $a_{i}^{*}$ can be evaluated as

$$
a_{i}^{*}=\frac{2}{\sqrt{\pi}} \int_{\theta_{i+1}}^{\theta_{i}} \exp \left(-t^{2}\right) d t, \quad \theta_{i}=\frac{1}{2 \sqrt{p_{i}}},
$$


which illustrates the decay of the $a_{i}^{*}$ as $i$ increases. In fact,

$$
0<a_{i}^{*} \leq \frac{1}{8 \pi^{3 / 2} p_{i}^{2}} \exp \left(-\frac{1}{4 p_{i+1}}\right) \leq \frac{1}{45 p_{i}^{2}}
$$

An illustrative plot of the situation is given in the figure where, for $i=1,2, \ldots, N$, the intervals $\left(p_{i+1}-p_{i}\right)=i / n$ with $n=36$ and $N=40$. It illustrates, consistent with the theory, how the sum of exponentials approximations approach $\exp \left(-x^{1 / 2}\right)$ from below.

\section{Conclusions}

The importance of the Kohlrausch (stretched exponential) decay behaviour in practical situations has been acknowledged by various authors including Anderssen et al. (2004); Sabelko et al. (1999); Hou et al. (2011). Though they draw attention to the fact, citing Pollard (1946) as the source, that a Kohlrausch decay can be reinterpreted as a system of exponential decays, the possibility of using Pollard's result to construct approximation to the Kohlrausch function has not been examined.

In Karmakar and Samanta (2003), this mentioned reinterpretation is used to justify the approximation of Kohlrausch function like data by a sum of two or three exponentials. The authors found that the fluorescence relaxation (decay) behaviour of some room temperature ionic liquids can be approximated by the sum of two exponentials. Such results can be viewed as indirect validation of the approximability of a Kohlrausch function by a not too large number of terms of a Dirichlet series representation.

Nevertheless, as is implicit in the figure, the number of exponentials required to yield an accurate reconstruction of Kohlrausch function behaviour is likely to be quite high.

\section{REFERENCES}

Anderssen, R. S., A. R. Davies, and F. R. de Hoog (2008a). On the sensitivity of interconversion between relaxation and creep. Rheologica Acta 47, 159-167.

Anderssen, R. S., A. R. Davies, and F. R. de Hoog (2008b). On the Volterra integral equation relating creep and relaxation. Inverse Problems 24, 035009 (p. 13).

Anderssen, R. S., S. Husain, and R. J. Loy (2004). The Kohlrausch function: properties and applications. $\operatorname{ANZIAMJ}(E)$ 45, C800-C816.

Anderssen, R. S. and R. J. Loy (2011). Sums of exponentials representations for the Kohlrausch function. Preprint.

Bernstein, S. N. (1928). Sur les fonctions absolument monotones. Acta Mathematica 52, 1-66.

Feller, W. (1966). An Introduction to Probability Theory and its Applications, Volume II. John Wiley \& Sons, New York.

Grinshpan, A., M. Ismail, and D. Milligan (2000). Complete monotonicity and diesel fuel spray. Math. Intel. 22, 43-53.

Hou, Z., D. Fu, and Q.-H. Qin (2011). An exponential law for stretching-relaxation properties of bone piezovoltages. Inter. J. Solids Structures 48, 603-610.

Karmakar, R. and A. Samanta (2003). Dynamics of solvation of the fluorescent state of some electron donor-acceptor molecules in room temperature ionic liquids, [BMIM][(CF3SO2)(2)N] and [EMIM][(CF3SO2)(2)N]. J. Phys. Chem. A 107, 7340-7346.

Lindsey, C. and G. Patterson (1980). Detailed comparison of the Williams-Watts and Cole-Davidson functions. J. Chem. Phys. 73, 3348-3357.

Liu, Y. (1999). Calculation of discrete relaxation modulus and creep compliance. Rheol. Acta 38, 357364. 
Liu, Y. (2001a). A direct method for obtaining discrete relaxation spectra from creep data. Rheol. Acta 40, 256-260.

Liu, Y. (2001b). Approximation by Dirichlet series with nonnegative coefficients. J. Approx. Theory 112, 226-234.

Macdonald, J. (1997). Accurate fitting of emittance spectroscopy frequency-response data using the stretched exponential model. J. Non-Crystal. Solids 212, 95-116.

Macdonald, J. (2006). Surprising conductive- and dielectric-system dispersion differences and similarities for two Kohlrausch-related relaxation-time distributions. J. Phys-Condensed Matter 18, 629-644.

Montroll, E. and J. Bendler (1984). On Levy (or Stable) distributions and the Williams-Watts model of dielectric-relaxation. J. Stat. Phys. 34, 129-162.

Nikonov, A., A. R. Davies, and I. Emri (2005). The determination of creep and relaxation functions from a single experiment. J. Rheol. 49, 1193-1211.

Osborne, B. and R. Anderssen (2003). Single-kernel characterization principles and applications. Cereal Chem. 80, 613-622.

Pollard, H. (1946). The representation of $\exp \left(x^{\lambda}\right)$ as a Laplace integral. Bull. Amer. Math. Soc. 52, 908-910.

Sabelko, J., J. Ervin, and M. Gruebele (1999). Observation of strange kinetics in protein folding. PNAS 96, 6031-6036.

Widder, D. V. (1946). The Laplace Transform. Princeton University Press, Princeton. 